\title{
Nonlinear dynamics of COVID-19 pandemic: modeling, control, and future perspectives
}

\author{
J. A. Tenreiro Machado $\mathbb{i}$ · Jun Ma
}

Published online: 3 September 2020

(C) Springer Nature B.V. 2020

Throughout the history of humankind, we find innumerable records of pandemics and devastating diseases, such as, to mention but a few, the Black Death, in the fourteenth century, or the 1918 Spanish flu, that led to millions of deaths. With the scientific advances of the last century, public opinion feels that such events are no longer likely to occur, but that is clearly a false belief. Yet there is some truth in this, since science can help society overcome these grand challenges. Friedrich Nietzsche said, "Illness is a clumsy attempt to arrive at health: we must come to nature's aid with intellect." However, we can ask ourselves if we are prepared for such dramatic crisis. On March 18, 2015, Bill Gates delivered a premonition lecture entitled "We're not ready for the next epidemic. We're not ready for it. But we can get there." Indeed, the tools of mathematics, computer science, biology, and medicine can guide us in mitigating and overcoming diseases. This special issue is about overarching efforts to understand, interpret, and possibly predict a specific disease spreading.

By the end of 2002, the severe acute respiratory syndrome (SARS) outbreak emerged in China and had a

\section{J. A. Tenreiro Machado $(\bowtie)$}

Department of Electrical Engineering, Institute of Engineering, Polytechnic of Porto, Rua Dr. António Bernardino de Almeida, 431, 4249-015 Porto, Portugal e-mail: jtm@isep.ipp.pt

J. Ma (凶)

Department of Physics, Lanzhou University of Technology, Lanzhou 730050, China

e-mail: hyperchaos@163.com significant impact across the globe. SARS was a relatively rare disease, and at the end of the epidemic, in June 2003, the incidence was more than 8 thousand cases with a case fatality rate of $11 \%$.

More recently, by the end of 2019 , the new related virus strain severe acute respiratory syndrome coronavirus 2 (SARS-CoV-2) was recorded, in Wuhan, China. Similar symptoms appeared in other regions of the world without taking rapid caution before this period. During 2020, the virus caused the Coronavirus disease 2019 (COVID-19) pandemic and spread exponentially in all continents with a huge impact on the global society, economy, and everyday life. At the time of writing this preface, more than 25.6 million cases were reported across 213 countries and territories, resulting in more than 855,600 deaths. The infection fatality ratio for coronavirus cannot be accurately calculated. In February, the World Health Organization reported estimates of the infection fatality ratio of about $0.6 \%$.

As with most of previous infectious diseases, the diffusion of COVID-19 can be studied and its evolution may be predicted. Data available from all affected countries can be employed to study models and to design algorithms that can help further understand this pandemic. The societal value of these studies is formidable not only for the deeper understanding that can be gained on the propagation patterns but also for the implementation of correct prevention policies. 
Stochastic dependence on a variety of factors, from human activities to virus mutations and metastasis, can induce new waves of infections and transmissions. A full control of COVID-19 mainly depends on the rescue efforts of governments, public health institutions, and conscious isolation of all citizens, because random traveling and mobility induce unpredictable infections. It is important to explore the mechanisms of propagation, describe hidden patterns associated with the virus, and predict future scenarios.

The journal Nonlinear Dynamics is a key platform for sharing new ideas, concepts, and methods about this timely topic. The selected manuscripts touch on topics related to COVID-19 within the scope of Nonlinear Dynamics and highlight key aspects such as:

- Parametric or data-driven modeling approaches for the virus infection and propagation

- Development of methods and algorithm for predicting the virus spreading dynamics

- Multi-scale (spatial/temporal) pattern formation

- Dynamical analysis and comparisons with other virus infections

- Implementation of control strategies and prevention schemes for future pandemic cases

- New modeling approaches and epidemiological data analysis.
We selected a collection of high-quality papers with important scientific advances and a variety of methods useful for the progress toward a solution for the COVID-19. Having these ideas in mind, we hope that this special issue will be of interest for scholars and researchers, aimed at finding new techniques and new applications of this urgent and important problem in all aspects of human society.

We wish to thank the authors for their cooperation and timely work. Moreover, we thank also the Editorin-Chief of Nonlinear Dynamics, Prof. Walter Lacarbonara, who motivated us with his proposal for this Special Issue at the outset of the COVID-19 outbreak and supported us during its development.

Guest Editors of the Special Issue

J. Tenreiro Machado, Porto

Jun Ma, Lanzhou

Publisher's Note Springer Nature remains neutral with regard to jurisdictional claims in published maps and institutional affiliations. 